\title{
Lexis
}

Journal in English Lexicology

Book reviews | 2009

\section{Wilfrid ROTGÉ, Anglais. Le vocabulaire}

Paris, Hatier, Collection « Bescherelle », Juin 2008, 384 pages

\section{Christelle Exare}

\section{(2)enEdition}

\section{Journals}

\section{Édition électronique}

URL : http://journals.openedition.org/lexis/1052

DOI : $10.4000 /$ lexis. 1052

ISSN : 1951-6215

\section{Éditeur}

Université Jean Moulin - Lyon 3

\section{Référence électronique}

Christelle Exare, «Wilfrid Rotgé, Anglais. Le vocabulaire », Lexis [En ligne], Recensions, mis en ligne le 16 mai 2009, consulté le 23 septembre 2020. URL : http://journals.openedition.org/lexis/1052 ; DOI : https://doi.org/10.4000/lexis.1052

Ce document a été généré automatiquement le 23 septembre 2020.

\section{(c) (i) (9)}

Lexis is licensed under a Creative Commons Attribution-NonCommercial-NoDerivatives 4.0 International License. 


\title{
Wilfrid ROTGÉ, Anglais. Le vocabulaire
}

Paris, Hatier, Collection « Bescherelle », Juin 2008, 384 pages

\author{
Christelle Exare
}

\section{RÉFÉRENCE}

Wilfrid RотGÉ, Anglais. Le vocabulaire. Paris, Hatier, Collection « Bescherelle », Juin 2008.

ISBN : 978-2-218-92622-8, Prix : 10,50€-384 pages

1 Anglais. Le vocabulaire, conçu par Wilfrid Rotgé, s'inscrit dans une tradition bicentenaire, et participe au renouveau amorcé récemment par la collection Bescherelle de l'éditeur Hatier. Il complète, dans cette collection, quatre autres ouvrages récents de grammaire et de vocabulaire, écrits par Wilfrid Rotgé, seul ou en collaboration. Le lectorat visé n'est mentionné que de façon indirecte dans l'avant-propos. Ce «vocabulaire de l'anglais" s'adresse à ceux qui constatent que «plusieurs années d'apprentissage de l'anglais ne permettent pas toujours de comprendre et se faire comprendre en anglais » ( 3 , en gras dans le texte). L'objectif implicite est donc, après un constat d'échec, de proposer un remède. Il est alors aisé d'imaginer l'intérêt avec lequel un tel ouvrage peut être accueilli par les élèves du secondaire, en quête perpétuelle du sésame de l'apprentissage de l'anglais : l'outil ou le livre qui réussira là où le système scolaire ne réussit que partiellement, voire même, échoue. Ce manuel se présente donc comme un pari osé, au regard du nombre d'ouvrages de vocabulaire qui fleurissent sur le marché. La somme d'entrées est désignée d'emblée comme garante de la qualité du livre : « riche de plus de 6000 mots » et proposant « plus de 1000 énoncés types ", il promet d'offrir à l'utilisateur «tous les outils pour mieux communiquer en anglais, à l'oral comme à l'écrit » (3).

2 Après un sommaire de trois pages, le livre est divisé en trois grandes parties associées chacune à une couleur : bleu pour le "lexique thématique », orange pour le "guide de communication" et vert pour l'index. Le "lexique thématique» présente le vocabulaire de l'anglais en quinze chapitres qui regroupent au total 50 rubriques. Le découpage de la langue est peu original. Il est conforme à ce que l'institution préconise 
(Ministère de l'Education nationale : 24). Un double classement (par thèmes et par antonymes / synonymes) fait évoluer le lecteur de l'être humain (titre de la première rubrique), jusqu'à l'univers (titre de la dernière rubrique). Probablement pour faciliter la mémorisation, les termes anglais (noms, verbes et adjectifs) sont donnés dans la première colonne, suivis d'une ou deux traductions en français. Une troisième colonne présente "quand c'est pertinent, une locution, un mot de la même famille, un synonyme etc. » (4). Chacune des quinze parties est subdivisée en sous-parties. Ainsi le volet «Professional life» est-il divisé en trois rubriques: "Employment and unemployment ", "Inside a firm » and " $\mathrm{A}$ few jobs ». La deuxième partie, intitulée "guide de communication", propose un classement inspiré de l'observation de situations de communication. Des rubriques aussi variées que: "saluer», "prendre congé », "parler de l'âge ", "dire l'heure», "exprimer une certitude, une probabilité »(338-340), et «au restaurant ou dans un pub»(362) regroupent des énoncés types dont la variété rivalise avec l'authenticité. L'index de mots en français révèle en fin de parcours ce que le sommaire a passé sous silence. L'ouvrage est un guide lexical dont la frontière avec le grammatical est ténue. De nombreuses entrées relevant du domaine plus couramment associé à la grammaire, comme " be able to », " can /could", "présent simple/en V-ing" sont ainsi répertoriées en caractère gras dans l'index et renvoient aux pages du guide de communication. L'index, qui est une sorte de fourre-tout des erreurs généralement recensées chez les francophones, regroupe une liste conséquente d'expressions en « dire » (comme " dire que ça va »). Le lectorat visé est un public de non-spécialistes, enclin à approfondir ses connaissances grâce à un outil simple d'accès. Bâti sur le modèle de l'incontournable Mot et l'idée, Le vocabulaire de la collection Bescherelle va plus loin et est plus séduisant, à maints égards.

3 La démarche de Wilfrid Rotgé est traditionnelle, communicationnelle et rassurante. Les mises en contexte dans les nombreux dialogues de la deuxième partie permettent à l'utilisateur de construire son bagage lexical en situant les mots dans un registre et dans une situation de communication donnée. Dans sa quête de l'explicite et dans son caractère univoque, le recours systématique à la traduction est un point d'appui important. Les "focus grammaticaux», au prescriptivisme sécurisant, mettent en garde le lecteur contre quelques chausse-trapes de l'anglais. L'ordonnancement et la présentation du lexique sont conventionnels. La langue n'est ni pensée ni segmentée selon un mode original. Il s'agit au contraire d'une catégorisation des mots selon une tradition francophone de la didactique de l'anglais. Son choix des incontournables s'oppose à une tradition anglo-saxonne. À titre d'exemple, English Vocabulary in Use consacre deux parties à go, get et aux expressions associées (Redman : 46-49). W. Rotgé s'abstient. En revanche, il liste dans une rubrique indépendante les différentes valeurs des modaux et des locutions équivalentes, alors que S. Redman ne répertorie aucun modal dans son index. Là où English Vocabulary in Use propose sept chapitres sur l'affixation et sur la composition, le Bescherelle opte pour une démarche plus grammaticale que lexicale, en sériant des notions telles que "parler d'un événement passé », " rattacher un événement passé au présent », "situer un événement dans le présent ", "situer un événement dans l'avenir ».

4 Si la perspective est conventionnelle sur le fond, elle est originale dans la forme. Les trois atouts de cet ouvrage sont sa clarté, sa légèreté de ton et la place accordée à la dimension orale de la langue. Ce petit livre, dans son format de poche $(14 \mathrm{~cm} \mathrm{x} 17 \mathrm{~cm})$ est d'une louable limpidité. Il est facile d'utilisation. Les couleurs attrayantes et 
contrastives, le souci du détail, la mise en page impeccable et les innovations typographiques, avec des polices de caractères spécialement créées pour cet opus, expliquent en partie pourquoi cet ouvrage a été plébiscité par les élèves de notre classe de Seconde, section européenne. En tête de chaque rubrique, des documents authentiques, comme une œuvre graphique (Hogarth, 64), des citations (Albert Einstein et Benjamin Disraeli, 180), ou encore des dessins humoristiques (76) viennent agrémenter l'ensemble et séduire l'œil. Au grand plaisir de son lecteur, Wilfrid Rotgé imprime une touche d'humour à son inventaire lexical. Le ton est donné avec la citation de Groucho Marx dès le premier chapitre. Les dialogues de la deuxième partie, fort amusants en ce qu'ils sont une concaténation d'une dizaine d'expressions relevant du même champ lexical, sont autant de clins d'œil au lecteur, qui saura sans aucun doute les apprécier. Voici l'exemple de la page 244, «Décrire quelqu'un ou quelque chose »:

Mel: True, You're pretty, even beautiful, you're always elegant but you're not my

type.

Flo: What's wrong with my physique? I would so much like to appeal to you.

Mel: Well, you have a nice body, not too small, not too big. It's just right.

Flo: So what? You say I'm good-looking. You're not handsome. You're fat and heavy, not very tall, narrow-chested. You look like Santa Claus, in fact. And you still won't go out with me?

Mel: Well, you have a point there. But your description of my physique is not exactly flattering.

Flo: That doesn't bother me. Personally, I just find you charming and attractive.

5 Un ouvrage écrit par un linguiste et qui fait sourire son lecteur à chaque début de chapitre est un objet rare qui mérite d'être salué.

6 La plus grande audace de cet ouvrage réside sans nul doute dans l'importance accordée à la dimension orale de la langue dans ce manuel qui cherche à «aider à communiquer». L'avant-propos souligne qu'« un effort particulier a été fait sur la phonétique ». Cet effort est réel mais limité, probablement pour ne pas effrayer un lectorat de non-spécialistes. La liste des symboles phonétiques utilisés n'est pas exhaustive. Seuls sont reproduits les phonèmes qui paraissent peu déchiffrables par un francophone. Les voyelles sont celles de l'anglais britannique standard. Les mots compliqués sont transcrits. Toujours dans le but de donner toute sa place à l'oral, la couverture annonce "des compléments audio en accès gratuit sur le site www.bescherelle.com », qui évoquent ce que proposait Georges Fauré dans son Manuel pratique d'anglais parlé en 1975. L'utilisateur, après une rapide identification sur la toile, a accès à une quantité d'exercices en relation avec les quatre derniers ouvrages d'anglais de la collection Bescherelle. Le lecteur peut écouter des conversations, faire des exercices grammaticaux lacunaires, ou encore s'entraîner à la traduction. Les dialogues sont hyperarticulés, à un débit normal, voire lent, avec quelques bruits parasites parfois, pour plus d'authenticité. Trois commandes sont disponibles : «play », «pause » et "stop », ce qui permet d'écouter le modèle et de répéter, sans toutefois avoir la possibilité de s'enregistrer. Il convient alors de s'interroger sur les stratégies d'auto-correction qui permettront à l'utilisateur d'améliorer ses compétences de communication. Dans quelle mesure une activité d'écoute, aussi active et réflexive soitelle, peut-elle contribuer à améliorer ses productions de façon significative? Dans le cadre de l'apprentissage d'une langue seconde en milieu non naturel, il semble difficile d'évoluer seul, sans l'intervention d'un tiers, capable d'identifier les erreurs de perception et de donner des orientations pour corriger les erreurs de production. Cependant, nous reconnaissons à cet ouvrage une réelle volonté de promouvoir la 
dimension orale du lexique. La mise en œuvre est novatrice sans être effrayante. Le pari, s'il est osé, est globalement gagné.

7 Malgré tout, les qualités de l'ouvrage en sont les défauts. S'il est louable d'extraire l'essentiel et de privilégier la clarté, les choix éditoriaux, dans tout ce qu'ils ont d'arbitraire, nuisent à l'ensemble en ce qu'ils affectent les dimensions culturelle et phonétique de l'ouvrage. L'anglais décrit dans ce livre est l'anglais britannique, même si les équivalences orthographiques avec l'anglais américain sont données (118: travelling et traveling) ou apparaissent comme synonymes (92). Dans certains dialogues de la deuxième partie (audibles en ligne), deux variétés d'anglais sont proposées (britannique standard et américain), comme par exemple dans le dialogue numéro 10 reproduit plus haut. Les autres variétés d'anglais non standard ne sont pas proposées comme modèles, conformément à ce que suggèrent les deux drapeaux britannique et américain reproduits sur la couverture. D'autres références culturelles puisent dans un substrat surtout européen. Par exemple, une courte liste des spécialités culinaires britanniques (364) suggère de manière elliptique que les États-Unis n'ont pas de culture culinaire. La maitrise (en audition et en production) des dialogues proposés permettra éventuellement à l'utilisateur de survivre à Brighton ("comprendre et se faire comprendre »), mais pas nécessairement de passer une commande dans un fast food du Bronx.

Le vœu d'exhaustivité, en ce qu'il est annoncé et non réalisé, est certainement un point faible de l'ouvrage. Le sous-titre «A few jobs » (83) illustre la difficulté de la tâche du linguiste, contraint de faire des choix déchirants. Nous regrettons que les paramètres qui président à ces choix ne soient pas mentionnés dans l'avant-propos. Dans une culture anglo-saxonne aujourd'hui mondialisée et ouverte sur les services, il est regrettable de ne pas voir recensés davantage d'emplois tertiaires. Pourquoi choisir de faire figurer a hairdresser et a miner plutôt que a nurse ou encore PR ? Pourquoi ne pas inclure dans la liste des métiers le mot solicitor, dont la fréquence frappera tout arpenteur de rues britanniques? Des études lexicométriques ont-elles précédé la conception de l'ouvrage? Quel est l'inventaire lexical qui préside au choix des mots? S'agit-il des études menées par le Conseil de l'Europe pour dresser un cadre commun de référence ? A titre d'exemple, une requête rapide réalisée grâce à l'outil de recherche lexicométrique de BNC donne 3096 occurrences du terme solicitor et 394 occurrences du terme hairdresser. La partie purement lexicale de l'ouvrage nous semble moins complète que ce qu'offre Le vocabulaire de l'anglais contemporain de Robert et Nathan (258-268). Un classement alternatif du lexique selon la catégorie grammaticale aurait fait d'ailleurs apparaître une relative pauvreté numéraire des adjectifs. Par exemple, dans la rubrique "Le commerce et les services ", buy et sell sont mentionnés, mais pas cheap ou expensive. L'adjectif, qui permet la nuance et enrichit la langue, est le parent pauvre du Vocabulaire de l'anglais de Wilfrid Rotgé. En comparaison, le Vocabulaire de l'anglais contemporain chez Nathan lui accorde plus de place. Le Petit vocabulaire actuel, paru chez Ophrys, classe les mots en trois catégories grammaticales (noms, verbes et adjectifs), et propose un index qui est véritablement lexical et non grammatical.

Dans le champ de la phonétique aussi, les choix paraissent arbitraires. L'auteur semble avoir été pris entre son désir de donner toute sa place à la dimension orale de la langue, et les nécessaires contraintes éditoriales. Seules les transcriptions «qui posent problème » (4) sont indiquées. Même s'il est justifiable de ne transcrire souvent que la syllabe accentuée d'un mot (buffalo, 104), comment la division entre ce qui est simple et 
ce qui est difficile a-t-elle été déterminée? Donnons quelques exemples : edible (48) traduit par «mangeable" uniquement, n'est pas transcrit ; bridesmaid (66) non plus. Pour the Renaissance, seule est proposée la transcription [rI'neIsəns], sans que les (fréquentes) réalisations alternatives proposées par $E D P$ et $L D$ ne soient mentionnées. Le mot apple (43), que nos élèves prononcent souvent ['eIpəl] au risque d'être perçu comme able par un anglophone, n'est pas transcrit. Une transcription de claw (42) n'est pas donnée. L'erreur peut pourtant être phonologique, au pluriel, si le locuteur confond avec close (n.). Les noms jaw (20) ou encore port (122) (pour n'en citer que deux) ne sont pas transcrits non plus, alors que la réalisation des voyelles d'arrière de l'anglais est une difficulté majeure pour les francophones. Dans la même veine, un souci de simplification préside au choix de ranger dans une même catégorie accent primaire et secondaire : death penalty (187) a un accent principal sur chaque terme du composé, au lieu d'un accent primaire sur death et d'un accent secondaire sur penalty. La proéminence perçue, signalée ici par un accent droit précédant la syllabe, nous paraît moins lisible par des béotiens que dans le Vocabulaire de Robert et Nathan, qui lui préfère le souligné. Reste à savoir si la connaissance de l'accentuation et de la transcription des mots aide le néophyte à reconnaître et à produire, en sachant que l'aide en ligne (www.bescherelle.com) ne reproduit pas les mots isolément. Se pose également la question de savoir si un va-et-vient entre les transcriptions isolées et les symboles phonétiques de l'anglais standard (4) aide l'utilisateur «à mieux communiquer en anglais ", en sachant que la langue, dans sa propension à assimiler et à réduire, ne se laisse ni segmenter ni représenter aussi facilement.

Le dernier livre en date de Wilfrid Rotgé est emblématique d'un effort de rupture dans la continuité. Clair, drôle, novateur et pratique, il se veut également rassurant et conventionnel. Il s'adresse à un public large de scolaires ou d'autodidactes, initiés mais non confirmés. L'omission d'une mention B1 qui renverrait à un niveau évalué dans le Cadre Européen des langues est éloquente en ce sens. Si son inventaire lexical basé sur "l'observation des échanges de la vie quotidienne »(3) se révèle limité, l'ouvrage est bien plus que ce qu'il ne dit de lui-même. Succédané de manuel scolaire, il est tout à la fois lexique, grammaire descriptive et prescriptive, laboratoire de langue, dictionnaire de prononciation allégé, guide touristique, recension d'expressions idiomatiques, recueil de poèmes, etc. Ces multiples facettes font de l'ouvrage de Wilfrid Rotgé un digest du vocabulaire de l'anglais. À l'image de ce que propose une pharmacie anglosaxonne, Le vocabulaire de l'anglais offre une palette diversifiée de remèdes pour soigner les symptômes du mal décrit dans son avant-propos. Il reste au lecteur à se préoccuper de l'origine du mal. Il manque à cet ouvrage un mode opératoire qui explique comment l'utilisateur peut s'en saisir et l'utiliser pour acquérir des réflexes et pour apprendre à apprendre. La mise en ligne de ressources audio est une première réponse au problème de la fixation et de la mémorisation du lexique. Nous souhaitons qu'un travail régulier avec ce livre modèle permette à l'apprenant de procéder par mimétisme pour acquérir des réflexes de catégorisation et pour apprendre à apprendre, en créant par exemple ses propres listes de vocabulaire, basées sur sa propre observation des scènes de la vie quotidienne. 


\section{BIBLIOGRAPHIE}

FONTANE, G., FROMONOT, J. et I. LEGUY. Vocabulaire de l'anglais contemporain. Paris : Nathan, 2007.

FAURÉ, G. Manuel pratique de l'anglais parlé. Paris : Hachette, 1975.

HORN, A. et C. GOSSET. Petit vocabulaire actuel. Paris : Ophrys, 2008.

Ministère de l'Education nationale. Programmes et accompagnements. Anglais. Classes de seconde, première et terminale. CNDP, 2007.

REDMAN, S. English Vocabulary in Use. Cambridge: Cambridge University Press, 2002.

REY, J., C. BOUSCAREN et A. MOUNOLOU. Le mot et l'idée 2. Paris : Ophrys, 1991.

\section{AUTEURS}

\section{CHRISTELLE EXARE}

Christelle Exare est professeur agrégé d'anglais, en poste dans un lycée de Haute-Normandie. Chercheuse, elle s'intéresse à la correction phonétique de l'anglais des francophones. Elle se forme en phonétique appliquée à l'ILPGA (Université Paris 3).

christelle.exare[at]ac-rouen.fr 